\title{
Solar wind interaction with the Reiner Gamma crustal magnetic anomaly: Connecting source magnetization to surface weathering
}

\author{
Andrew R. Poppe ${ }^{\mathrm{a}, \mathrm{b}, *}$, Shahab Fatemi ${ }^{\mathrm{a}, \mathrm{b}}$, Ian Garrick-Bethell ${ }^{\mathrm{c}, \mathrm{d}}$, Doug Hemingway ${ }^{\mathrm{c}, \mathrm{e}}$, Mats Holmström ${ }^{\mathrm{f}}$ \\ ${ }^{a}$ Space Sciences Laboratory, 7 Gauss Way, University of California at Berkeley, Berkeley, CA, USA \\ ${ }^{b}$ Solar System Exploration Research Virtual Institute, NASA Ames Research Center, Mountain View, CA, USA \\ ${ }^{c}$ Dept. of Earth and Planetary Science, University of California at Santa Cruz, Santa Cruz, CA, USA \\ ${ }^{d}$ School of Space Research, Kyung Hee University, Yongin-si, Gyeonggi-do 446-701, Republic of Korea \\ ${ }^{e}$ Miller Institute for Basic Research in Science, University of California at Berkeley, Berkeley, CA, USA \\ ${ }^{f}$ Swedish Institute of Space Physics, Kiruna, Sweden
}

\begin{abstract}
Remanent magnetization has long been known to exist in the lunar crust, yet both the detailed topology and ultimate origin(s) of these fields remains uncertain. Some crustal magnetic fields coincide with surface albedo anomalies, known as lunar swirls, which are thought to be formed by differential surface weathering of the regolith underlying crustal fields due to deflection of incident solar wind protons. Here, we present results from a three-dimensional, self-consistent, plasma hybrid model of the solar wind interaction with two different possible source magnetizations for the Reiner Gamma anomaly. We characterize the plasma interaction with these fields and the resulting spatial distribution of charged-particle weathering of the surface and compare these results to optical albedo measurements of Reiner Gamma. The model results constrain the proposed source magnetizations for Reiner Gamma and suggest that vertical crustal magnetic fields are required to produce the observed "dark lanes."

Keywords: Moon, surface, Magnetic fields, Solar wind
\end{abstract}

\section{Introduction}

One of the most fundamental open questions in lunar science is the origin of remanent magnetic anomalies in the lunar crust. These fields are highly non-dipolar, sporadic in location about the Moon, and can exceed hundreds of nT in some locations [Mitchell et al., 2008]. Crustal fields were first detected during the Apollo era [Dyal et al., 1974; Hood et al., 1981], with comprehensive measurements of the global distribution of crustal magnetization made later by the Lunar Prospector mission [Lin et al., 1998; Halekas et al., 2001; Mitchell et al., 2008]. Several theories have been proposed for the origin of these crustal fields, including shock remanent magnetization (SRM) of impact ejecta [e.g., Crawford and Schultz, 1988; Gattacceca et al.,

* Corresponding author

Email address: poppe@ssl.berkeley.edu (Andrew R. Poppe) 
2010], amplification of SRM at basin antipodal points due to converging magnetic fields and impact ejecta [Hood et al., 2001; Hood and Artemieva, 2008], and thermoremanent magnetization (TRM) of impact ejecta cooling in an early lunar dynamo field [e.g., Collinson, 1993; Wieczorek et al., 2012]. The relative fitness of these theories has implications for both the evolution of the lunar dynamo [e.g., Garrick-Bethell et al., 2009] and impact-induced magnetization throughout the solar system [e.g., Girdler et al., 1992; Collinson, 1993; Connerney et al., 2004]. Understanding the distribution and topology of crustal fields and their underlying magnetization sources is critical to differentiating between these various theories at different anomalies.

Several lunar crustal anomalies are also correlated with sinuous, surficial albedo anomalies on the lunar regolith, known as "lunar swirls" [e.g., El-Baz, 1972]. Seemingly unique in the solar system [see Blewett et al., 2010, 2014], lunar swirls have prompted several formation theories to explain the correlated presence of crustal magnetic fields and albedo anomalies. These have included the possibility of the emplacement of magnetic fields and the deposition of light-colored material during cometary impact [Schultz and Srnka, 1980], the retardation of space weathering and darkening of soils due to deflection of solar wind protons in highly-magnetized regions [Hood and Schubert, 1980; Hood and Williams, 1989], and magnetic sorting of sub-micron dust grains with differing spectral properties due to complex electromagnetic field geometries within anomalies [Garrick-Bethell et al., 2011]. Support for the solar wind shielding hypothesis of Hood and Schubert [1980] is found from several recent results from the Lunar Reconnaissance Orbiter (LRO) and Chandrayaan missions. These findings include that swirls are indistinguishable at decimeter depth scales from surrounding non-swirl material (which argues against cometary impact) [Neish et al., 2011], that swirls show indications of reduced space weathering yet are thermophysically identical to non-swirl regions [Glotch et al., 2015], and that swirls are relatively deficient in hydroxyl (OH) signatures compared to nonswirl regions [Kramer et al., 2011b,a]. The latter evidence supports the solar wind shielding hypothesis since hydroxyl is presumably formed in lunar regolith due to bombarding solar wind protons reacting with regolith oxygen [Pieters et al., 2009; Sunshine et al., 2009].

Reiner Gamma, located in the western Oceanus Procellarum, is the archetypical example of both a magnetic anomaly and a lunar swirl. Figure 1(a) shows an image from the Lunar Reconnaissance Orbiter Camera (LROC) [Robinson et al., 2010] of the main portion of Reiner Gamma. The distinctive nature of the bright lobes separated by two narrow dark lanes has been previously noted and used as a guide for proposing an underlying source magnetization [Hemingway and Garrick-Bethell, 2012]. Using the solar wind shielding hypothesis, Hemingway and Garrick-Bethell [2012] argued that the bright regions of Reiner Gamma seen in Figure 1 indicated the presence of horizontal magnetic fields, as this topology has been shown to be more effective at shielding solar wind protons than vertically-oriented fields [e.g., Wang et al., 2013]. The proposed source magnetization consisted of two approximately parallel rows of dipoles situated under the bright lobes just outside the "dark lanes" of Reiner Gamma, with moments oriented parallel to the surface. This magnetization, while inherently non-unique, nevertheless matched Lunar Prospector 
observations at altitudes of $18 \mathrm{~km}$ and above. In this paper, we test both the proposed source magnetization from Hemingway and Garrick-Bethell [2012] and a similar, yet alternate magnetization to quantify the selfconsistent plasma interaction of these fields with the solar wind. In turn, we examine the charged particle surface weathering within the anomaly and compare to both the magnetic topology and optical albedo images of Reiner Gamma for both cases. We describe the model in Section 2 and compare results between the two different magnetizations in Section 3. We discuss the implications of our results with respect to the possible sub-surface magnetization sources for Reiner Gamma and space weathering processes in Section 4.

\section{Model Description}

\subsection{Plasma hybrid model}

In order to model the solar wind interaction with the Reiner Gamma magnetic anomaly, we have employed a three-dimensional, self-consistent plasma hybrid model. This model has previously been used to study lunar plasma interactions both at the global scale [Holmström et al., 2012; Fatemi et al., 2014; Poppe et al., 2014] and on regional scales [Fatemi et al., 2015]. The hybrid model tracks particle ions at realistic proton mass and fluid electrons while solving Maxwell's equations. Plasma is injected from the upstream boundary with specified density, temperature, and flow speed, and is removed when striking the lunar surface. As the hybrid model does not treat electrons as particles but rather as a fluid, we do not include photoelectrons from the surface, nor do we include the accumulation of surface charge from the solar wind plasma. This approximation is reasonable for modeling the scale sizes of magnetic anomalies, as typical photoelectron Debye lengths are on the order of meters [Poppe and Horányi, 2010], while the magnetic anomaly is on the order of kilometers. Lunar crustal magnetic fields are included in the model and are self-consistently solved along with the interplanetary magnetic field (IMF). The model coordinate system is such that $\hat{x}$ is anti-parallel to the solar wind flow and $\hat{y}$ and $\hat{z}$ are both parallel to the surface. The model domain is $x=[-50,350] \mathrm{km}, y=[-480,480] \mathrm{km}$, and $z=[120,120] \mathrm{km}$, with $10 \mathrm{~km}$ grid cell size and 16 particles per cell. The $\hat{y}$ and $\hat{z}$ directions are fully periodic for both particles and fields, while in the $\hat{x}$ direction, particles are injected at the upper boundary with a specific distribution and are absorbed at the lunar surface. For all runs presented here, we used standard solar wind parameters: flow speed of $350 \mathrm{~km} / \mathrm{s}$ at normal incidence $\left(\approx 640 \mathrm{eV}\right.$ proton energy), density $6.0 \mathrm{~cm}^{-3}$, electron and ion temperatures of 4.3 and $8.6 \mathrm{eV}$, respectively, $\mathrm{IMF} B_{y}=-5 \mathrm{nT}$, and solar wind dynamic pressure, $P=1.22 \mathrm{nPa}$.

Reiner Gamma presents a difficult application to model because of both its size and inferred magnetic topology. The hybrid model grid cell size must be larger than the electron inertial length (here, $d_{e}=c / \omega_{p e} \approx$ $3 \mathrm{~km}$, where $c$ is the speed of light and $\omega_{p e}$ is the electron plasma frequency); thus, we set our grid size to $10 \mathrm{~km}$, however, this would only barely resolve the anomaly, which has a characteristic length of $\approx 30$ $\mathrm{km}$ across the main swirl (see Figure 1(a)). Furthermore, the anticipated small-scale magnetic fields [i.e., 
Hemingway and Garrick-Bethell, 2012] are expected to have gradients at or below this minimum hybrid cell size. Therefore, in order to proceed, we made two approximations: first, we focused our model only on a cross-section of the main, central portion of the Reiner Gamma anomaly and swirl, shown in Figure 1(a) within the dashed rectangle. Hemingway and Garrick-Bethell [2012] have postulated that this portion of the anomaly can be represented by a source magnetization consisting of two approximately parallel lines of buried dipoles; thus, our model consists of two parallel lines running symmetrically along one entire axis of the hybrid model domain (see red dashed lines in Figure 1(b) and section 2.2 below). Secondly, in order to fully resolve the plasma interaction within the hybrid model, we have enlarged the modeled anomaly by a factor of approximately five, i.e., the dipole lines in the hybrid model are separated horizontally by 80 $\mathrm{km}$ compared to $\approx 15 \mathrm{~km}$ as postulated by Hemingway and Garrick-Bethell [2012] for Reiner Gamma. This approximation still preserves the fundamental plasma interaction scale lengths at Reiner Gamma as the electrons in the model are magnetized $\left(r_{e}<0.2 \mathrm{~km}\right.$ at $20 \mathrm{~km}$ altitude) and the protons are unmagnetized $\left(r_{i}>45 \mathrm{~km}\right.$ at $20 \mathrm{~km}$ altitude), where $r_{e}$ and $r_{i}$ are the electron and ion gyroradii, respectively.

\subsection{Source magnetization model}

As discussed in Section 2.1, the magnetic fields for the Reiner Gamma anomaly are assumed to be generated from two parallel, infinite line magnetizations. The vector magnetic field, B, from a buried line of dipoles is given by [Hemingway and Garrick-Bethell, 2012],

$$
\mathbf{B}(\mathbf{r})=\frac{\mu_{o}}{4 \pi r^{4}}\left[4\left(\mathbf{m}^{\prime} \cdot \mathbf{r}\right) \mathbf{r}-2 \mathbf{m}^{\prime} r^{2}\right]
$$

where $\mathbf{m}^{\prime}$ is the magnetization per unit length and $\mathbf{r}$ is the position vector. In addition to the source magnetization suggested by Hemingway and Garrick-Bethell [2012] for Reiner Gamma, where all dipoles are oriented in the $+\hat{y}$ direction (horizontal to the surface), we ran the hybrid model for several alternate arrangements of buried dipoles with respect to their depth, magnitude, and orientation. By visually comparing the modeled plasma flux to the surface with the surface albedo from Figure 1(a) for each trial, we found an alternate arrangement with the dipole lines vertically anti-aligned (Case 2, described below) that matched well with the albedo patterns. Importantly, we note the inherent non-uniqueness of both of these models, and the extensive parameter space available for the source magnetization with respect to dipole depth, separation, and orientation. Nevertheless, these two cases provide a useful comparison for the role of the magnetic topology in determining the plasma interaction and flux to the surface.

Figure 1(c-h) shows the two crustal magnetic field geometries used for simulating Reiner Gamma. For Case 1, shown in the lower left panels of Figure 1, we placed two lines of dipoles $5 \mathrm{~km}$ below the surface, separated by $80 \mathrm{~km}$, with $\left|m^{\prime}\right|=10^{8} \mathrm{~A} \cdot \mathrm{m}$. The magnetization direction of both lines for Case 1 is horizontal (parallel to the surface), shown in the bottom of panel 1(e) as the two arrows. This geometry is taken from Hemingway and Garrick-Bethell [2012]. Panels 1(c) and (d) show the magnetic field components and 
magnitude across the anomaly at $80 \mathrm{~km}$ and $20 \mathrm{~km}$ altitude, respectively, while panel 1(e) shows the field lines. At $80 \mathrm{~km}$ altitude, the field magnitude has a peak at approximately $9 \mathrm{nT}$, with a sinusoidally varying vertical field and a horizontal field with a single maximum. At $20 \mathrm{~km}$, the field is more complex, with two separate maxima in the magnitude and horizontal component and a higher-order varying vertical field.

Case 2, shown in the lower right panels of Figure 1, has two dipoles similarly placed $5 \mathrm{~km}$ below the surface and $80 \mathrm{~km}$ apart, but with magnetization directions oriented vertically opposite (i.e., one line of dipoles points upwards and the other points downwards). The line magnetization magnitude is also $\left|\mathrm{m}^{\prime}\right|=10^{8}$ A.m. At $80 \mathrm{~km}$, panel 1(f), the fields are qualitatively similar to those at $80 \mathrm{~km}$ for Case 1, with a single dominant horizontal maximum and a sinusoidal vertical component. At $20 \mathrm{~km}$ altitude, however, the field structure for Case 2 is significantly different, with two sharp maxima in the vertical field at $\pm 40 \mathrm{~km}$ and a somewhat weaker set of maxima in the horizontal component. Importantly, while we have chosen two different source magnetizations for Cases 1 and 2, the field geometries at high altitudes $(80 \mathrm{~km}$ for this simulation, i.e., the separation distance of the dipoles) are similar. This results from the non-uniqueness of fitting magnetic source distributions, yet ensures that our alternative source magnetization qualitatively agrees with Lunar Prospector magnetometer observations of Reiner Gamma to first-order, keeping in mind our enlarged simulation relative to the Reiner Gamma anomaly.

\section{Model Results}

Figure 2 shows the hybrid simulation results for Case 1 and Case 2, respectively. For Case 1, the six panels show (a) the total magnetic field magnitude (solar wind plus crustal field), (b) the electrostatic potential (relative to 0 Volts at $x=+\infty$ ), (c) the electric field magnitude, (d) the solar wind density, (e) the solar wind proton energy spectrum at the surface, and (f) the solar wind proton flux (black, normalized to the flux in the un-magnetized region) and the electrostatic potential at the surface (red), respectively. The strongest magnetic fields occur directly over the buried dipole lines, with a maximum strength above the surface of $\approx 620 \mathrm{nT}$, comparable to that estimated from orbital magnetometer measurements [Tsunakawa et al., 2015]. As shown by numerous other simulations and observations [e.g., Poppe et al., 2012; Saito et al., 2012; Futaana et al., 2013; Jarvinen et al., 2014; Deca et al., 2014; Fatemi et al., 2015], crustal field interaction with the solar wind induces a strong electrostatic potential above the anomaly as electrons are magnetically prevented from entering the anomaly region while protons, with larger gyroradii, directly penetrate the anomaly region and induce both ambipolar and Hall electric fields. For Case 1, the maximum electrostatic potential reaches more than $+400 \mathrm{~V}$, or $\approx 60 \%$ of the incident solar wind proton energy. Commensurate with these potentials, panel 2 (e) shows that the solar wind protons are strongly decelerated from $600 \mathrm{eV}$ to less than $100 \mathrm{eV}$ directly over the buried dipoles. Far from the dipole $(|y|>75 \mathrm{~km})$, the protons impact the surface at the undisturbed solar wind energy, while protons in between the dipole lines $(|y|<30 \mathrm{~km})$ are 
decelerated by $\approx 150 \mathrm{eV}$. The deceleration and deflection of the solar wind protons above the dipole lines reduces the proton flux to the surface down to $\approx 40 \%$ of the solar wind flux, while slightly enhancing the flux to the surface both between and just outside the two dipole lines, shown in panel 2(f). The electrostatic potential at the surface, drawn in red in panel 2(f), strongly anti-correlates with the surface flux since the electric field is responsible for reflecting/deflecting the protons (and not the magnetic field). Thus, for this configuration of the dipoles (both horizontal), the surface is shielded in two narrow regions directly overhead the dipoles, while experiencing slightly elevated fluxes within and just outside of the dipoles (see Section 3.1 for further discussion).

In comparison, Figure $2(\mathrm{~g}-\mathrm{l})$ shows the results of the plasma simulation with the dipoles vertically antialigned, Case 2. The magnetic field strength reaches a maximum of $\approx 710 \mathrm{nT}$ at the surface in two regions immediately above the dipoles (similar to Case 1). In contrast to the electrostatic potential from Case 1 (panel 2(b)), the potential for Case 2 has a single lobe reaching $+500 \mathrm{~V}$ in between the two dipole lines and two smaller lobes just outside each dipole line at approximately $+350 \mathrm{~V}$ maximum. The strong potential in the center of Case 2 is due to the strong horizontal magnetic field component at this location, which induces relatively strong electric fields through the Hall term in the generalized Ohm's Law $(E \propto J \times B)$. The dominance of the Hall term over other terms in the generalized Ohm's Law (convective, ambipolar) has been seen in previous hybrid model simulations of solar wind interactions with lunar magnetic anomalies [Jarvinen et al., 2014; Fatemi et al., 2015]. The solar wind proton flux to the surface also differs from Case 1, with proton deceleration to approximately $200 \mathrm{eV}$ just outside the dipole lines $(70<|y|<100 \mathrm{~km}$ ), sharp increases in the flux at the undisturbed solar wind energy at $|y| \approx 50 \mathrm{~km}$, and broad shielding and deceleration of the solar wind flux in between the dipole lines $(|y|<40 \mathrm{~km})$. We note in particular the sharpness of the two peaks in the solar wind flux to the surface immediately over the vertically-oriented dipoles in this case; these features are caused by deflection of protons from the neighboring shielded regions and may be responsible for the fine-grained "dark lanes" in the surface albedo of Reiner Gamma (see Figure 1(a-b) and section 3.1). These narrow lanes of access to the surface should be contrasted with Case 1, where Figure 2(f) shows only broad proton access to the surface and two narrow regions of surface shielding.

\subsection{Comparison to albedo features}

For the two source magnetization geometries presented here, we can compare the resulting surface weathering patterns, Figures 2(f) and 2(l), respectively, to the surface albedo observations from LROC, Figure 1(b). Specifically, we computed the mean surface brightness from the LROC image along the long axis of the modeled region outlined by the rectangle in Figure 1(a). Figure 1(b) shows this average with the two dark lanes and three bright lobes labeled. We take the inverse of the net proton flux to the surface from our model as a proxy for the relative brightness of the surface; however, we note importantly that the relative and respective roles of charged particle bombardment and micrometeorite impacts on the regolith weath- 
ering process remain an open question. It is possible that charged particle bombardment is the dominant weathering agent for the lunar regolith due to faster weathering timescales [Loeffler et al., 2009; Hemingway et al., 2015] or it is also possible that charged particle bombardment is a necessary precursor to surface weathering by micrometeorite impacts, and that a lack of charged particle bombardment induces a slower rate of weathering by micrometeorites [e.g., Blewett et al., 2011; Glotch et al., 2015, and references therein]. Despite this uncertainty, the relative proton flux to the surface as derived from the model can serve as an indicator of local space weathering.

Figure 3 compares the relative brightness of the modeled surface for Cases 1 and 2, respectively, normalized to the relative brightness outside the magnetized region as a function of the dipole separation distance (80 km in the model). Figure 3 also shows the angle of the magnetic field with respect to the surface at 30 $\mathrm{km}$ altitude in blue. For Case 1, shown in panel 3(a), the surface brightness is characterized by two bright lanes immediately over the buried dipoles $(x= \pm 1$, vertical dashed lines) and slightly darker regions just outside of and in between the bright lanes. In contrast, the surface brightness for Case 2, panel 3(b), is roughly the inverse of Case 1, with two dark lanes over the buried dipoles and bright lobes outside of and in between the dark lanes. A comparison with the relative brightness of the LROC image of Reiner Gamma, Figure 1(b), demonstrates that Case 2, with the oppositely-oriented vertical dipole lines under the surface, qualitatively reproduces the observed surface brightness. In both cases, the angle between the magnetic field and the surface strongly anti-correlates with the modeled surface brightness within \pm 2 dipole separations (i.e., where the field is strong). Strongly horizontal fields shield the surface via induced electric fields (at least for the noon-time case run here), producing bright regions, while strongly vertical fields correspond to dark lanes, where the electric field is weak and solar wind protons easily penetrate the anomaly down to the surface. We do note that the incident angle of the plasma (i.e., approximately the solar zenith angle) may change the overall plasma interaction with anomalies and thus, a full understanding of how anomalies shield the surface requires study of non-noontime cases. While these cases are not presented in this work, they are identified as a subject of future research. Additionally, we note that there may be an effective lower limit to the relative brightness of the lunar surface under enhanced charged particle flux. While Figure 3(b) would suggest that the "dark lanes" in the swirl would appear darker than the surrounding unmagnetized regolith, it is possible that there is a saturation level to surface darkening. This could be interpreted as support for the idea that charged particle weathering is a necessary, but not sufficient process for surface darkening, and that micrometeoroid bombardment is the additional, necessary process required to darken protonated regolith [see discussion in Blewett et al., 2011]. 


\section{Conclusion}

We have simulated the solar wind interaction with lunar crustal magnetic fields using two hypothetical source magnetizations for the Reiner Gamma anomaly in an effort to connect the source magnetization to the surface weathering patterns. Our simulations reinforce the conclusion that plasma fluxes to the lunar surface are controlled by the topology of the crustal magnetic field, namely, that horizontally dominant regions provide more shielding than vertically dominant regions through the generation of strong, vertical electric fields [i.e., Jarvinen et al., 2014; Fatemi et al., 2015]. As demonstrated by the results of Case 2, vertical cusps in the magnetic field topology can allow the undisturbed solar wind to penetrate directly to the surface, although we note that magnetic mirroring of solar wind electrons within cusp regions (i.e., vertically oriented fields) may generate additional ambipolar electric fields that are not captured in the hybrid simulation here [Poppe et al., 2012]. Thus, if the solar wind shielding hypothesis for the generation of lunar swirls is correct [Hood and Schubert, 1980], then plasma simulations of the type presented here can constrain the near-surface magnetic field topology of crustal magnetic anomalies by correlating modeled surface plasma precipitation maps with surface albedo.

As an application of this conclusion, we note that while the observed magnetic fields above the Reiner Gamma anomaly can be most easily explained by a bulk horizontal magnetization [Kurata et al., 2005; Nicholas et al., 2007; Hemingway and Garrick-Bethell, 2012], our simulations show that purely horizontal magnetization does not yield the desired surface weathering pattern, i.e., panel 3(a). In contrast, the vertical magnetization of Case 2 does qualitatively reproduce the observed surface weathering pattern, which suggests that some strongly vertically magnetized source may be present with the Reiner Gamma anomaly. Thus, higher order structure to the magnetization source proposed by Hemingway and GarrickBethell [2012] should be considered for Reiner Gamma. Possible mechanisms for generating such smaller magnetization signatures on top of the dominant horizontal signature include: vertical deformation of a uniformly horizontally magnetized material via tectonic mechanisms (i.e., the formation of lunar graben or rilles), impact cratering and re-arrangement of the magnetized source material before the emplacement of the mare basalts (although impacts can de-magnetize the rock), and emplacement of higher-order field components via shock-remanent magnetization and convergence of antipodal fields [Hood and Artemieva, 2008]. One could also consider that the magnetized source material for Reiner Gamma may be composed of neighboring thin slabs with varying heights, reflecting the stochastic nature of ejecta deposition. Such slabs can produce broadly horizontal field structure with sharp vertical components near the slab edges [see Connerney et al., 2004, Fig. 5]. A further investigation of possible source magnetizations and correlations between the modeled surface plasma flux and observed albedo features for Reiner Gamma and other magnetic anomalies is clearly warranted.

While these simulations have highlighted the increasing ability of plasma simulations to connect source 
magnetization profiles to surface weathering, there are several avenues for continued investigation with respect to the self-consistent plasma interaction, including (1) the effect of solar zenith angle variations in determining proton access to the surface (noting that most of the solar wind flux to the surface over a lunar day occurs within $30^{\circ}$ of noon), (2) the role of solar wind and IMF variability in determining both the plasma interaction and surface access (i.e., see Fatemi et al. [2015] for a study on solar wind pressure variability at the Gerasimovich anomaly), and (3) the presence of higher order field components on the solar wind interaction near the surface. Ultimately, in-situ measurements of both magnetic fields and plasma within lunar crustal magnetic anomalies [Garrick-Bethell et al., 2013] are required to fully understand the nature of anomalies and the various processes involved in space weathering.

\section{Acknowledgements}

A.R.P. and S.F gratefully acknowledge support from NASA's Solar System Exploration Research Virtual Institute, grant \#NNX14AG16A. This publication is SSERVI contribution \#SSERVI-2015-160. The authors acknowledge the International Space Science Institute (ISSI) for hosting a workshop series that in part inspired this work as well as two reviewers for constructive and helpful comments. The software used in this work was in part developed by the DOE NNSA-ASC OASCR Flash Center at the University of Chicago. This research was conducted using resources provided by the Swedish National Infrastructure for Computing (SNIC) at the High Performance Computing Center North (HPC2N), Umeå University, Sweden. The LROC data are publicly available from the NASA PDS Imaging Node (http://pds-imaging.jpl.nasa.gov/). I. G-B. was partially supported by the BK21 PLUS program through the National Research Foundation (NRF), funded by the Ministry of Education of Korea. 
Blewett, D. T., Buczkowski, D. L., Ruesch, O., Scully, J. E., O’Brien, D. P., Gaskell, R., Roatsch, T., Bowling, T. J., Ermakov, A., Hiesinger, H., Williams, D. A., Raymond, C. A., Russell, C. T., 2014. Vesta's north pole quadrangle Av-1 (Albana): Geologic map and the nature of the south polar basin antipodes. Icarus 244, 13-22.

Blewett, D. T., Coman, E. I., Hawke, B. R., Gillis-Davis, J. J., Purucker, M. E., Hughes, C. G., 2011. Lunar swirls: Examining crustal magnetic anomalies and space weathering trends. J. Geophys. Res. 116 (E02002).

Blewett, D. T., Denevi, B. W., Robinson, M. S., Ernst, C. M., Purucker, M. E., Solomon, S. C., 2010. The apparent lack of lunar-like swirls on Mercury: Implications for the formation of lunar swirls and for the agent of space weathering. Icarus 209, 239-246.

Collinson, D. W., 1993. Magnetism of the Moon - a lunar core dynamo or impact magnetization? Surveys in Geophysics 14, $89-118$.

Connerney, J. E. P., Acuña, M. H., Ness, N. F., Spohn, T., Schubert, G., 2004. Mars Crustal Magnetism. Space Sci. Rev. 111, 1-32.

Crawford, D. A., Schultz, P. H., 1988. Laboratory observations of impact-generated magnetic fields. Nature 336, 50-52.

Deca, J., Divin, A., Lapenta, G., Lembège, B., Markidis, S., Horányi, M., 2014. Electromagnetic Particle-in-Cell Simulations of the Solar Wind Interaction with Lunar Magnetic Anomalies. Phys. Rev. Lett. 112 (151102).

Dyal, P., Parkin, C. W., Daily, W. D., 1974. Magnetism and the Interior of the Moon. Rev. Geophys. Space Phys. 12 (4), $568-591$.

El-Baz, F., 1972. The Alhazen to Abul Wafa Swirl Belt: An extensive field of light-colored sinuous markings. NASA Spec. Publ. 315, 29-93.

Fatemi, S., Holmström, M., Futaana, Y., Lue, C., Collier, M. R., Barabash, S., Stenberg, G., 2014. Effects of protons reflected by lunar crustal magnetic fields on the global lunar plasma environment. J. Geophys. Res.: Space Physics 119.

Fatemi, S., Lue, C., Holmström, M., Poppe, A. R., Wieser, M., Barabash, S., Delory, G. T., 2015. Solar wind plasma interaction with Gerasimovich lunar magnetic anomaly. J. Geophys. Res.: Space Physics 120.

Futaana, Y., Barabash, S., Wieser, M., Lue, C., Wurz, P., Vorburger, A., Bhardwaj, A., Asamura, K., 2013. Remote energetic neutral atom imaging of electric potential over a lunar magnetic anomaly. Geophys. Res. Lett. 40, $262-266$.

Garrick-Bethell, I., ad H. Sanchez, R. P. L., Jaroux, B. A., Bester, M., Brown, P., Cosgrove, D., Dougherty, M. K., Halekas, J. S., Hemingway, D., Lozano, P. C., Martel, F., Whitlock, C. W., 2013. Lunar magnetic field measurements with a cubesat. In: SPIE Defense, Security, and Sensing. International Society for Optics and Photonics.

Garrick-Bethell, I., Head III, J. W., Pieters, C. M., 2011. Spectral properties, magnetic fields, and dust transport at lunar swirls. Icarus 212, 480-492.

Garrick-Bethell, I., Weiss, B. P., Shuster, D. L., Buz, J., 2009. Early Lunar Magnetism. Science 323, $356-359$.

Gattacceca, J., Boustie, M., Hood, L., Cuq-Lelandais, J., Fuller, M., Bezaeva, N. S., de Resseguier, T., Berthe, L., 2010. Can the lunar crust be magnetized by shock: Experimental groundtruth. Earth Plan. Sci. Lett. 299, 42-53.

Girdler, R. W., Taylor, P. T., Frawley, J. J., 1992. A possible impact origin for the Bangui magnetic anomaly (Central Africa). Tectonophysics 212, 45-58.

Glotch, T. D., Bandfield, J. L., Lucey, P. G., Hayne, P. O., Greenhagen, B. T., Arnold, J. A., Ghent, R. R., Paige, D. A., 2015. Formation of lunar swirls by magnetic field standoff of the solar wind. Nature Comm. 6 .

Halekas, J. S., Mitchell, D. L., Lin, R. P., Frey, S., Hood, L. L., Acuña, M. H., Binder, A. B., Nov. 2001. Mapping of crustal magnetic anomalies on the lunar near side by the Lunar Prospector electron reflectometer. J. Geophys. Res. 106 (E11), $27,841-27,852$.

Hemingway, D., Garrick-Bethell, I., 2012. Magnetic field direction and lunar swirl morphology: Insights from Airy and Reiner Gamma. J. Geophys. Res. 117 (E10012).

Hemingway, D. J., Garrick-Bethell, I., Kreslavsky, M. A., 2015. Latitudinal variation in spectral properties of the lunar maria 
and implications for space weathering. Icarus 261, 66-79

Holmström, M., Fatemi, S., Futaana, Y., Nilsson, H., 2012. The interaction between the Moon and the solar wind. Earth Planets Space 64, 237-245.

Hood, L. L., Artemieva, N. A., 2008. Antipodal effects of lunar basin-forming impacts: Initial 3D simulations and comparisons with observations. Icarus 193, 485-502

Hood, L. L., Russell, C. T., Coleman Jr., P. J., 1981. Contour Maps of Lunar Remanent Magnetic Fields. J. Geophys. Res. 86 (B2), 1055-1069.

Hood, L. L., Schubert, G., 1980. Lunar Magnetic Anomalies and Surface Optical Properties. Science 208, 49-51.

Hood, L. L., Williams, C. R., 1989. The Lunar Swirls: Distribution and Possible Origins. Proc. 19th Lunar Sci. Conf. 19, $99-113$.

Hood, L. L., Zakharian, A., Halekas, J., Mitchell, D. L., Lin, R. P., Acuña, M. H., Binder, A. B., Nov. 2001. Initial mapping and interpretation of lunar crustal magnetic anomalies using Lunar Prospector magnetometer data. J. Geophys. Res. 106 (E11), $27,825-27,839$.

Jarvinen, R., Alho, M., Kallio, E., Wurz, P., Barabash, S., Futaana, Y., 2014. On vertical electric fields at lunar magnetic anomalies. Geophys. Res. Lett. 41.

Kramer, G. Y., Besse, S., Dhingra, D., Nettles, J., Klima, R., Garrick-Bethell, I., Clark, R. N., Combe, J.-P., III, J. W. H., Taylor, L. A., Pieters, C. M., Boardman, J., McCord, T. B., 2011a. $\mathrm{M}^{3}$ spectral analysis of lunar swirls and the link between optical maturation and surface hydroxyl formation at magnetic anomalies. J. Geophys. Res. 116 (E00G18).

Kramer, G. Y., Combe, J.-P., Harnett, E. M., Hawke, B. R., Noble, S. K., Blewett, D. T., McCord, T. B., Giguere, T. A., 2011b. Characterization of lunar swirls at Mare Ingenii: A model for space weathering at magnetic anomalies. J. Geophys. Res. 116 (E04008).

Kurata, M., Tsunakawa, H., Saito, Y., Shibuya, H., Matsushima, M., Shimizu, H., 2005. Mini-magnetosphere over the Reiner Gamma magnetic anomaly region on the Moon. Geophys. Res. Lett. 32 (L24205).

Lin, R. P., Mitchell, D. L., Curtis, D. W., Anderson, K. A., Carlson, C. W., McFadden, J., Acuña, M. H., Hood, L. L., Binder, A. B., Sep 1998. Lunar Surface Magnetic Fields and Their Interaction with the Solar Wind: Results from Lunar Prospector. Science 281, 1480-1484.

Loeffler, M. J., Dukes, C. A., Baragiola, R. A., 2009. Irradiation of olivine by $4 \mathrm{keV} \mathrm{He}^{+}$: Simulation of space weathering by the solar wind. J. Geophys. Res. 114 (E03003).

Mitchell, D. L., Halekas, J. S., Lin, R. P., Frey, S., Hood, L. L., Acuña, M. H., Binder, A., 2008. Global mapping of lunar crustal magnetic fields by Lunar Prospector. Icarus 194, 401-409.

Neish, C. D., Blewett, D. T., Bussey, D. B. J., Lawrence, S. J., Mechtley, M., Thomson, B. J., The Mini-RF Team, 2011. The surficial nature of lunar swirls as revealed by the Mini-RF instrument. Icarus 215, 186-196.

Nicholas, J. B., Purucker, M. E., Sabaka, T. J., 2007. Age spot or youthful marking: Origin of Reiner Gamma. Geophys. Res. Lett. 34 (L02205).

Pieters, C. M., et al., Oct. 2009. Character and Spatial Distribution of $\mathrm{OH} / \mathrm{H}_{2} \mathrm{O}$ on the Surface of the Moon Seen by $\mathrm{M}^{3}$ on Chandrayaan-1. Science 326, 568-572.

Poppe, A., Horányi, M., 2010. Simulations of the Photoelectron Sheath and Dust Levitation on the Lunar Surface. J. Geophys. Res. 115 (A08106).

Poppe, A. R., Fatemi, S., Halekas, J. S., Holmström, M., Delory, G. T., 2014. ARTEMIS observations of extreme diamagnetic fields in the lunar wake. Geophys. Res. Lett. 41.

Poppe, A. R., Halekas, J. S., Delory, G. T., Farrell, W. M., 2012. Particle-in-cell simulations of the solar wind interactions with lunar crustal magnetic anomalies: Magnetic cusp regions. J. Geophys. Res. 117 (A09105).

Robinson, M. S., et al., 2010. Lunar Reconnaissance Orbiter Camera (LROC) Instrument Overview. Space Sci. Rev. 150, 
Saito, Y., Nishino, M. N., Fujimoto, M., Yamamoto, T., Yokota, S., Tsunakawa, H., Shibuya, H., Matsushima, M., Shimizu, H., Takahashi, F., 2012. Simultaneous Observation of the Electron Acceleration and Ion Deceleration over Lunar Magnetic Anomalies. Earth Planets Space 64, 83-92.

Schultz, P. H., Srnka, L. J., 1980. Cometary collisions on the Moon and Mercury. Nature 284, 22-26.

Sunshine, J. M., Farnham, T. L., Feaga, L. M., Groussin, O., Merlin, F., Millikan, R. E., A’Hearn, M. F., 2009. Temporal and Spatial Variability of Lunar Hydration As Observed by the Deep Impact Spacecraft. Science 326, 565-568.

Tsunakawa, H., Takahashi, F., Shimizu, H., Shibuya, H., Matsushima, M., 2015. Surface vector mapping of magnetic anomalies over the Moon using Kaguya and Lunar Prospector observations. J. Geophys. Res.: Planets 120, 1160-1185.

Wang, X., Howes, C. T., Horányi, M., Robertson, S., 2013. Electric potentials in magnetic dipole fields normal and oblique to a surface in plasma: Understanding the solar wind interaction with lunar magnetic anomalies. Geophys. Res. Lett. 40, 1686-1690.

Wieczorek, M. A., Weiss, B. P., Stewart, S. T., 2012. An Impactor Origin for Lunar Magnetic Anomalies. Science 335, 12121215. 

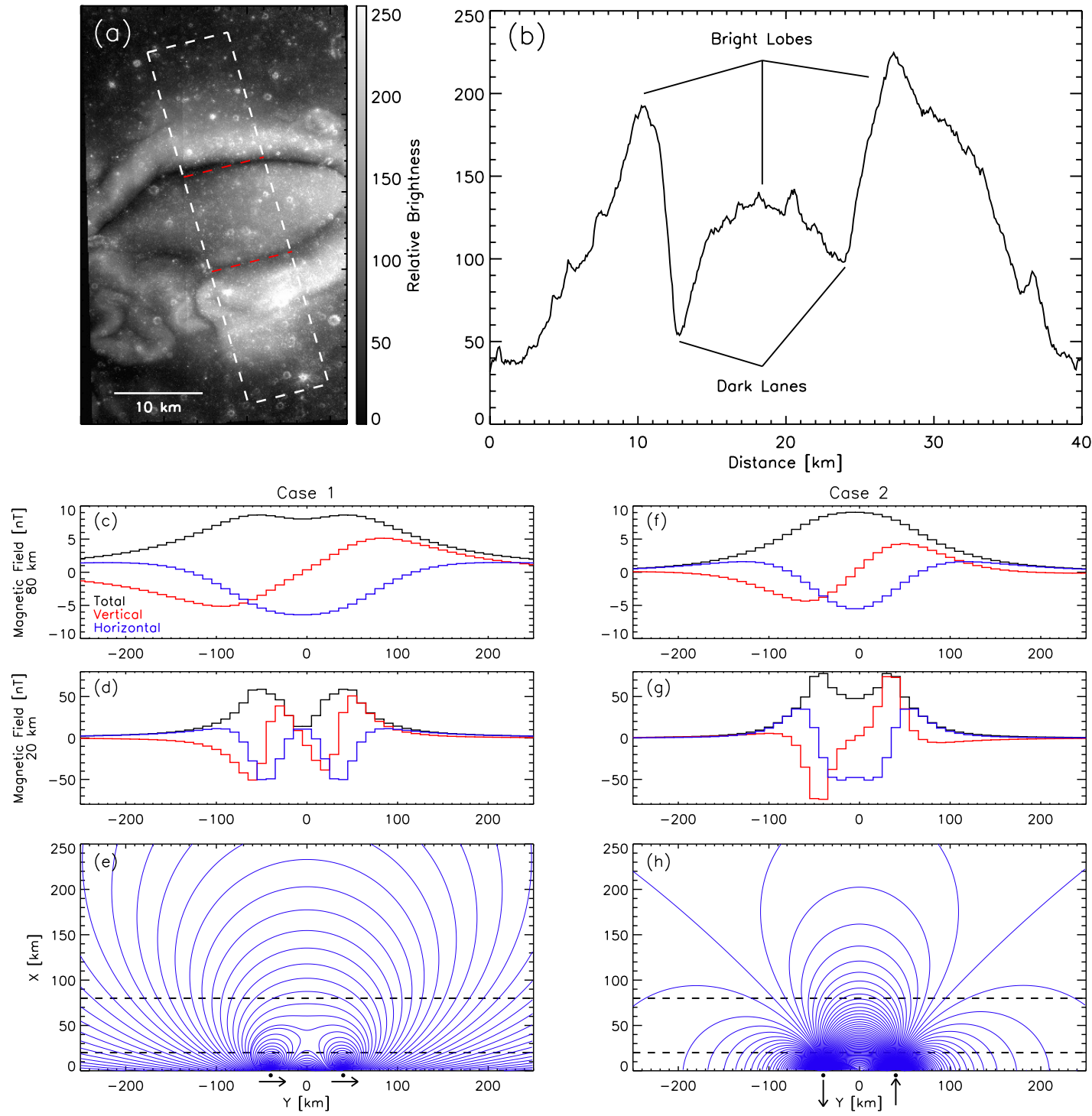

Figure 1: (a) Image NAC_ROI_REINRGAMHIA_E075N3009_20M of the Reiner Gamma swirl from the LROC instrument on-board LRO [Robinson et al., 2010]. The dashed rectangle denotes the region of interest modeled here and the red dashed lines depict the location and orientation of the modeled dipole lines. (b) The mean relative brightness along the long axis of the rectangle from panel (a), with bright lobes and dark lanes denoted. (c-f) A depiction of the source magnetizations investigated in Cases 1 and 2, respectively. The upper panels show the undisturbed magnetic field trace at $80 \mathrm{~km}$ altitude, the middle panels show the undisturbed magnetic field trace at $20 \mathrm{~km}$ altitude, and the lower panels show the field lines. Arrows underneath panels (c) and (f) denote the direction of magnetization for the buried lines of dipoles, as described in the text. 



-- Case 2 ---
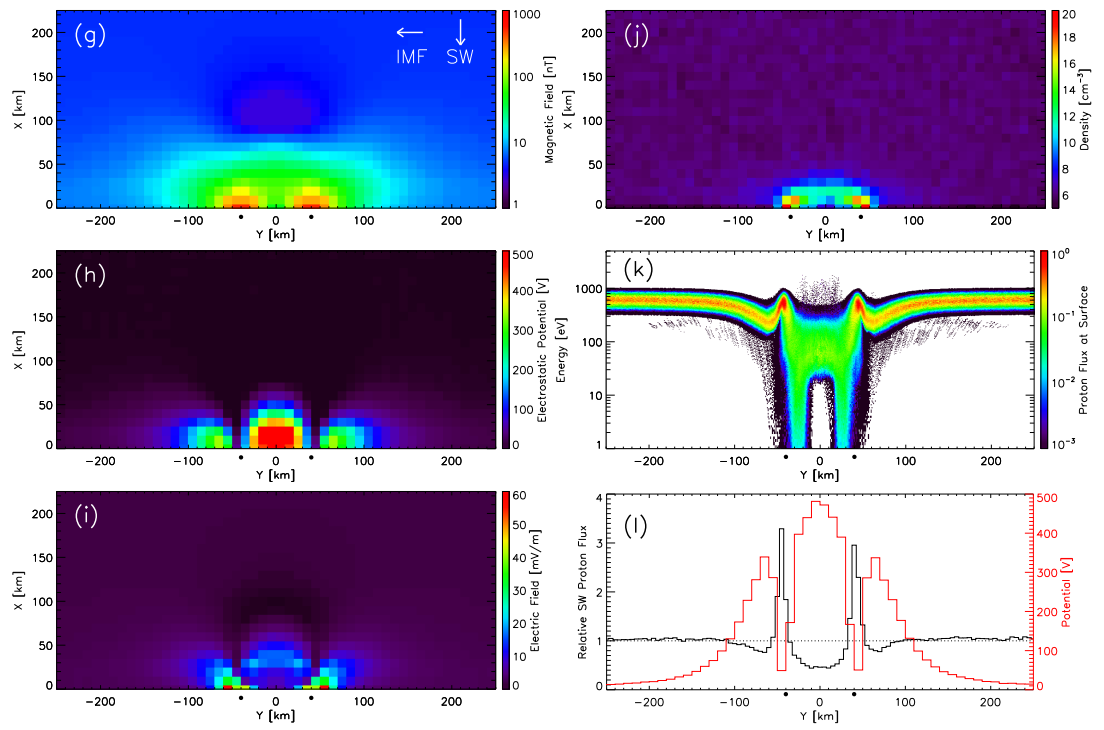

Figure 2: Hybrid model results for the Case 1 configuration: (a) the total magnetic field magnitude, (b) the electrostatic potential, (c) the magnitude of the electric field, (d) the solar wind proton density, (e) the solar wind proton energy spectrum at the surface, and (f) the relative solar wind proton flux to the surface. The pair of dots underneath each panel denote the position of the dipole lines. Panels (g)-(l) are the same format for Case 2. 

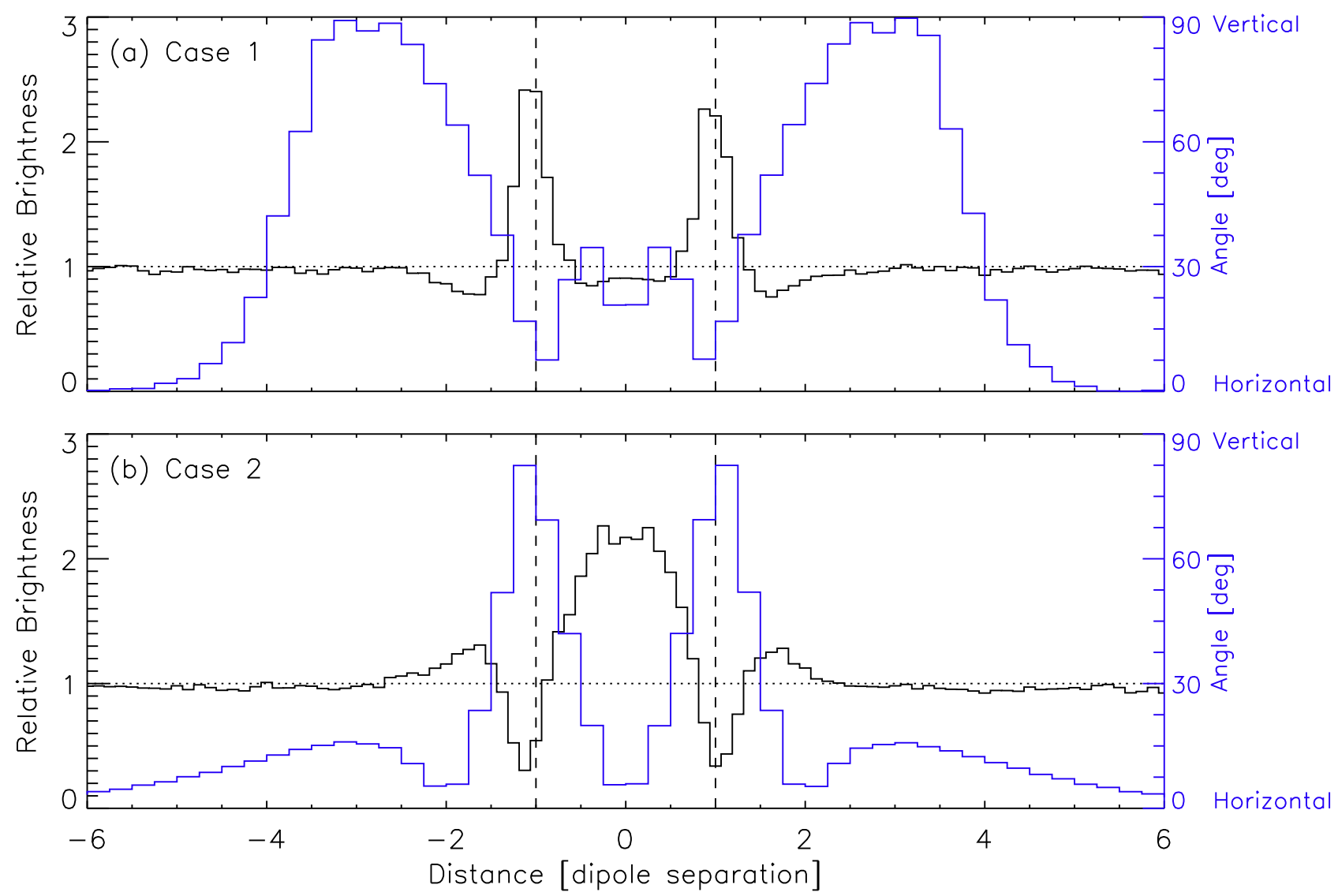

Figure 3: A comparison of the modeled relative surface brightness (black) and the angle between the magnetic field and the surface at $30 \mathrm{~km}$ altitude (blue) for (a) Case 1 and (b) Case 2, respectively. Vertical dashed lines denote the location of the buried dipole lines and the horizontal dotted line marks the un-shielded relative surface brightness, for reference. 